\title{
Chatic Dynamics of ECG Waveforms at Global and Microscopic Scales: Theory and Applications
}

\author{
Dmitriy Melkonian $^{* 1}$, Edward Barin ${ }^{2}$ and Homayoun Bahramali ${ }^{3}$ \\ ${ }^{I}$ Western Clinical School, University of Sydney, Sydney, NSW 2006, Australia \\ ${ }^{2}$ Cardiac Rhythm Management Unit, Department of Cardiology, Royal North Shore Hospital, Sydney, NSW 2065. \\ Australia \\ ${ }^{3}$ Bioemotec, Sydney, NSW 2006, Australia
}

\begin{abstract}
A theory is developed for describing the electrocardiogram (ECG) in terms of underlying cellular processes of ion transport. ECG evolvement over time is regarded as a sequence of partly overlapping self-similar transient potentials, with the generic mass potential (GMP) being the basic element. Using equations of the nonhomogeneous birth-and-death process (BDP), a particle model of GMP in the form of chaotic BDP is deduced. The formalism of deterministic chaos not only brings together the deterministic and stochastic factors underlying ECG genesis, but also does this with the minimum number of free parameters. No matter how complex the system of underlying ion transport, just a single parameter (the chaos factor), yet directly derived from microscopic scale equations, aggregates essential aspects of the ECG dynamics. This paradigm is investigated in numerical experiments, and qualified as the chaotic transformations effect. At the global level the mass effect of chaotic transformations is described by a system of nonlinear differential equations. Applications of this theory are supported by the method of high-resolution fragmentary decomposition which resolves component temporal overlap and reconciles the ECG waveforms with the dynamics of chaotic processes. This technique goes beyond conventional measures such as ECG peak amplitudes and latencies, and provides a more comprehensive analysis of the dynamics of ECG waveforms. In particular, the resolution of the component overlap provides means for recognising the complex composition of $\mathrm{Q}, \mathrm{R}$ and $\mathrm{S}$ waves, and co-operative action of the systems producing $\mathrm{R}$ and $\mathrm{S}$ waves.
\end{abstract}

Keywords: ECG, multi-scale-model, chaos, nonhomogeneous BDP, fragmentary decomposition, generic mass potential, Fourier analysis, SBF algorithm.

\section{INTRODUCTION}

The surface electrocardiogram (ECG) obtained by recording the potential differences between electrodes placed on the surface of the skin is widely accepted as an objective marker of the heart control mechanisms under normal and pathological conditions. Conventional conceptualization of the ECG as an ensemble of P, Q, R, S and T major waves, associates these deflections in ECG with different aspects of heart performance during the cardiac cycle. Quantitative analysis of ECG waveforms is essential for the identification of cardiac diseases including myocardial ischemia, myocardial infarction, bundle branch block, ventricular hypertrophy, atrial and ventricular arrhythmia, etc. The ECG can also indicate the presence of generalized disorders that affect the rest of the body.

However, interpretation of ECG waveforms is largely empirical, and there have been many efforts to create testable analytical models of ECG generation from cellular sources. The mechanisms involved in electrical activity of the heart are organized spatially in a hierarchical fashion from elements of diverse nature. Accordingly, numerous models

*Address correspondence to this author at the Western Clinical School, University of Sydney, Sydney, NSW 2006, Australia; Tel: 621 0296182228;

E-mails: dmitri@med.usyd.edu.au,dmitri@bioemotec.com of heart activity include a variety of levels of description and implement different modeling approaches [1]. The most fundamental advances in modeling of electrical phenomena have been achieved using the formalism of the HodgkinHuxley equations of the squid giant axon as a mathematical framework for creation of cardiac cell models [2]. The major developments in this field were recently reviewed by Winslow and colleagues [3, 4]. They showed that a number of models have reached a high degree of physiological detail in an attempt to link the machinery of voltage-gated membrane currents with the dynamics of the action potential produced by the heart muscle cell.

The wide interest in the models of the heart muscle cell in the search of advanced tools of ECG quantitative analysis arises from the proposal that model myocyte can be used as a "building block" for establishment of structure-function relationships between the parameters of transmembrane ionic channels and conventional ECG parameters [5-7]. However, there are two major problems inherent in the integration of cellular sources into the global models.

The first problem is how to deal appropriately with modeling complications posed by the multiplicity of cellular elements and uncertainty surrounding the character of their interactions. Remarkable progress has been achieved in the significant increase of the number of cellular elements which 
can be modeled in numerical simulations. However, an unambiguous physical and physiological interpretation of these results demands solution of the second problem.

The second problem is the absence of a consensus on the basis model. The two leading computational models of ventricular cells differ widely in dynamic assumptions, physiological and anatomical detail $[8,9]$. The extent to which the models are in contradiction is uncertain. A meta analysis of these models in the context of their re-use as subcomponents of multiscale biophysical systems exposed "significant differences in representations of model components together with a number of unresolved issues surrounding the description of model construction and the link with experimental data" [10]. The authors conclude that none of the models subjected to the meta analysis is superior, and declare the necessity of new modeling approaches.

Yet the investigation of similar theoretical problems in connection with the component composition of event related potential (ERP) put forward a model of the mass potential as a composite of self-similar geometric waveforms, with the GMP as a basic element [11]. Subsequent developments have been supported by advanced method of fragmentary decomposition which combines identification of GMPs with the resolution of component temporal overlap [12]. The method has proven to be a powerful tool for analysis of the eyeblink electromyogram (EMG) [13]. Functional neuroanatomy of the cellular sources of this complex oscillatory potential is quite different from the neural sources of ERPs. Despite these differences, the fragmentary decompositions of ERPs and eyeblink EMGs into ensembles of generic mass potentials produced remarkably similar results.

In our attempts to explain this unexpected situation in mathematical terms, we focused our attention on the fact that GMP represents a specific combination of two Gaussian functions. In view of the ubiquity of the Gaussian distribution, this study has been motivated by the idea that rather than being an oversimplification, the analytical form of GMP provides the key insight into the physics of mass potentials, and suggests a constructive framework for development of pertinent theory which involves questions of probability and deterministic chaos. To our knowledge, this study is the first to treat the emergence of ECG waveforms from elementary sources using concepts and methods of the deterministic chaos.

\section{THEORY}

\section{Physical Basis}

It is generally accepted that the ECG is produced by electrical activity of the heart muscle cells. Since the electrodes for ECG recordings are distant from the heart, the ECG reflects some peculiarities of an electrical field surrounding the heart.

Quantitative analysis of the field is complicated by the unobservability of all relevant variables. Every theory of the ECG is therefore based on simplifications which determine the field under idealized conditions.
One of the most fruitful simplifications comes from the fact that boundaries of the intracellular space, the cell membranes, have high resistance, compared with resistance of the extracellular space. This experimental fact implies that little current from extracellular sources flows through the cell membrane. Hence, the intracellular and extracellular electric transients are governed by different physical factors. A membrane potential transient that takes place uniformly over the entire membrane surface of a cell would generate no extracellular current flow and would therefore generate no extracellular potential transient. A nonuniformity of the membrane potential creates a loop of current flow which arises from a set of current sources embedded in an extracellular medium.

The shape and inhomogeneity of the volume conductor may also influence the recorded extracellular potential transient. However, these factors are usually neglected in the calculations of extracellular potentials. It is generally accepted that within the range of frequencies of physiological interest, capacitive, inductive, magnetic, and propagative properties of the extracellular medium can be neglected [14]. In other words, the extracellular space is regarded as a three-dimensional resistive medium.

The simplest model of how the extracellular current sources create a dipole is indicated schematically in Fig. (1). The large circles in (a) and (b) show the membrane as a closed surface which separates the extracellular space from intracellular medium. One might imagine that this separating material is in some way sewed together from the membranes of individual cells, and thus controls movements of ions from intracellular space to extracellular space and vice versa according to the general rules of the membrane transport. (a)

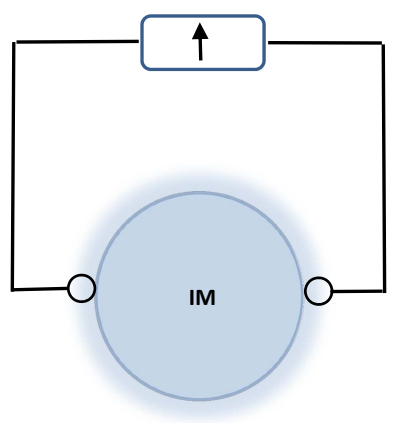

(b)

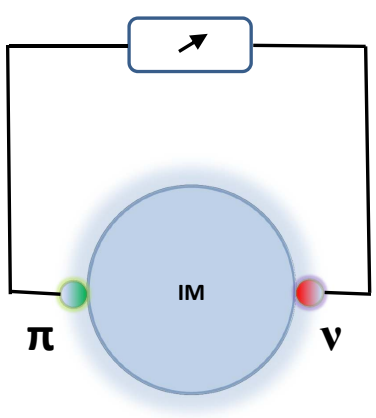

Fig. (1). Schematic representation of an idealized membrane that separates intracellular medium (IM) of an ensemble of heart cells from the extracellular space. The shaded areas show the extracellular layers of charged particles. (a) A uniform distribution of charged particles. (b) Under the non-uniformity in the distribution of charged particles, the volume elements act as a source and a sink of an electrical dipole.

The sources of electricity are ions, i.e. charged particles, which cross the cell membrane in both directions during all phases of the heart activity. It is realistic to regard a thin layer uniformly distributed over the entire exterior membrane surface as the container of extracellular charged particles. Let us divide this layer into small volume elements 
of equal size. Two of such compartments are shown in (a) and (b) of Fig. (1) by the small circles.

Since the transport of ions across the membrane is governed by probabilistic rules, it is natural to describe the particle populations in stochastic terms. All random variables will be written in boldface letters. Given particular compartment, let integer-valued random variables $\mathbf{N}_{+}(t)$ and $\mathbf{N}_{-}(t)$ measure at the time $t$ the numbers of elementary positive and negative charges, respectively. From the point of view of a distant point in the extracellular medium, the compartment can be envisaged as a positive charge if $\mathbf{N}_{+}(t)>\mathbf{N}_{-}(t)$, or as a negative charge if $\mathbf{N}_{+}(t)<\mathbf{N}_{-}(t)$. The summary charge of the compartment is proportional to the integer-valued random variable $\mathbf{X}(t)=\mathbf{N}_{+}(t)-\mathbf{N}_{-}(t)$.

A uniform distribution of charged particles that is characterized by practically equal values of $\mathbf{X}(t)$ for different compartments does not create a significant potential difference between the two compartments, as it is shown in Fig. (1a). In this situation the galvanometer does not indicate an extracellular current.

A condition that is necessary to create potential differences in the extracellular space is the non-uniformity in the distribution of charged particles close to the external surface of the membrane. This non-uniformity may take different forms. In our theory, we associate creation of a dipole with synchronous activation of a large number of cells in local conductance volumes. Such regions of the nonuniformities are depicted in Fig. (1b) as the current source $(\pi)$ and current sink $(v)$ which are attached from the two different sites to the membrane. The current source is associated with a positive charge the size of which is measured by integer valued random variable $\mathbf{X}_{\pi}(t)$. The current sink is associated with a negative charge described in a similar way by $\mathbf{X}_{v}(t)$. Given resistive external medium, the voltage created by the dipole is $\mathbf{V}(t) \propto\left[\mathbf{X}_{\pi}(t)-\mathbf{X}_{v}(t)\right]$. This form of the field potential is quite general, and may be used as a starting point for stochastic modelling of different conditions. We wish to work out in mathematical detail the ionic machinery of the GMP generation.

\section{Generic Mass Potential}

Based on a series of papers concerned with the dynamics of mass potentials [11-13], we regard the GMP as a model of a monolithic waveform of the mass potential. The GMP is economically defined by the set of the three parameters, $\kappa, \psi$ and $\chi$, in the form

$$
\mathrm{g}(t)=\kappa \cdot \operatorname{bud}[(t-\psi) / \chi]
$$

where

$$
\operatorname{bud}(x)=\frac{1}{\sqrt{2 \pi}}\left(\mathrm{e}^{-(x-1)^{2} / 2}-\mathrm{e}^{-(x+1)^{2} / 2}\right) \cdot 1(x)
$$

is a standard GMP. In this formula $1(x)$ is a unit step function,

$$
1(x)=\left\{\begin{array}{l}
1 \text { for } x \geq 0 \\
0 \text { for } x<0
\end{array}\right.
$$

Being illustrated by Fig. (2), the standard GMP has a steeply rising left flank and a slowly decreasing right flank. The peak value indicated by the vertical dotted line corresponds to $\mathrm{x} \approx 1.2$ at which bud(1.2) $\approx 0.356$.

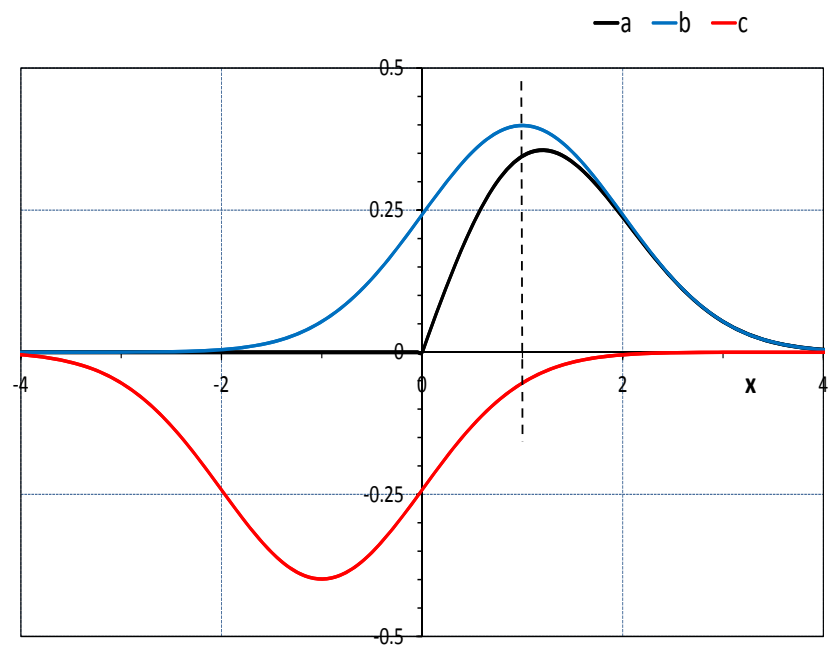

Fig. (2). Shows shifted positive (b) and negative (c) Gaussian functions the sum of which gives at $\mathrm{x}>0$ the standard GMP (a).

\section{Non-Homogeneous BDP}

The equation (1) may be given a physical basis in terms of the source and sink if we present GMP in the form

$\mathrm{g}(t)=\frac{1}{\sqrt{2 \pi}}\left[\mathrm{g}_{\pi}(t)-\mathrm{g}_{\mathrm{v}}(t)\right] \cdot 1(t)$,

where

$\mathrm{g}_{\pi}(t)=\mathrm{e}^{-\frac{(t-\chi)^{2}}{2 \chi^{2}}}, \quad \mathrm{~g}_{\mathrm{v}}(t)=\mathrm{e}^{-\frac{(t+\chi)^{2}}{2 \chi^{2}}}$,

and subscripts " $\pi$ " and " $v$ " indicate the source and sink as the origins of the corresponding terms of $g(t)$. The unit step function attributes the beginning of the transient to the $\mathrm{t}=0$.

Given a deterministic character of GMP, we associate $g_{\pi}(t)$ and $g_{v}(t)$ with expected values of the random variables $\mathbf{X}_{\pi}(t)$ and $\mathbf{X}_{v}(t)$, i.e.

$\mathrm{g}_{\pi}(t)=\mathrm{E}\left\{\mathbf{X}_{\pi}(t)\right\}$ and $\mathrm{g}_{\mathrm{v}}(t)=\mathrm{E}\left\{\mathbf{X}_{v}(t)\right\}$,

where $\mathrm{E}\{\}$ denotes a mathematical expectation.

A general framework within which we will describe $\mathbf{X}_{\pi}(t)$ and $\mathbf{X}_{v}(t)$ is adopted from previous modelling studies of the short-term synaptic plasticity in which the "birth-and-death" process has been settled as a tool for the analysis of transmembrane particle transfers [15]. The idea is to regard the membrane as a border that divides the population of relevant particles into the two compartments. A particle is allowed to leave or enter the 
compartment through the membrane. Such event is regarded as the "death" of a particle in the primary compartment, and simultaneous "birth" of a particle in the target compartment. Given particular compartment in Fig. (1b), we regard the "birth" as an admission of a particle to the compartment from the cell interior. The "death" is regarded as removal of a particle from the compartment.

We choose as an adequate mathematical framework a nonhomogeneous birth-and-death process in which the birth and death rates are arbitrary functions of time [16]. Let the integer-valued time-dependent random variable $\mathbf{X}(\mathrm{t})$ measure at time $t$ the size of the population. In the general case, the mean of a non-homogeneous BDP is [16]

$\mathrm{m}(t)=\mathrm{E}\{\mathbf{X}(t)\}=\mathrm{e}^{-\rho(t)}$,

where

$\rho(t)=\int_{0}^{t}[\mu(\tau)-\lambda(\tau)] d \tau$,

$\mu(\tau)$ and $\lambda(\tau)$ are the rates of the death and birth, respectively.

\section{Chaotic "Birth-and-Death" Processes}

The formalism of a non-homogeneous BDP allows us to treat the creation of the dipole using both stochastic and deterministic factors. We introduce two specific types of non-homogenous processes termed a "chaotic birth-anddeath process" and "chaotic death process". Both processes are specified by the parameter $\chi$ named a "chaos factor" or " $\chi$ factor".

We introduce the chaotic birth-and-death process $\{\lambda, \mu(t), \chi\}$ as the model of the particle population in the source compartment. The process is defined at $t \geq 0$ by the following rates of the birth and death of particles:

$\lambda_{\pi}=1 / \chi, \quad \mu_{\pi}(t)=t / \chi^{2}$.

The mean of the chaotic process is

$\mathrm{m}_{\pi}(t)=\mathrm{E}\left\{\mathbf{X}_{\pi}(t)\right\}=\mathrm{e}^{-\rho_{\pi}(t)}$,

where

$\rho_{\pi}(\mathrm{t})=\frac{t^{2}-2 t \chi}{2 \chi^{2}}$

With regard to (3), it is easily deduced that

$\mathrm{g}_{\pi}(t)=\mathrm{m}_{\pi}(t) / \sqrt{e}$.

We define the chaotic death process $\{\mu(t), \chi\}$ as the model of the particle population in the sink compartment. The process is defined at $t \geq 0$ by the following rate of the death of particles:

$\mu_{v}(t)=(t+\chi) / \chi^{2}$.

The mean of $\mathbf{X}_{v}(t)$ is

$\mathrm{m}_{v}(t)=\mathrm{E}\left\{\mathbf{X}_{v}(t)\right\}=\mathrm{e}^{-\rho_{v}(t)}$, where

$\rho_{v}(\mathrm{t})=\frac{t^{2}+2 t \chi}{2 \chi^{2}}$.

Comparison with (3) shows that

$\mathrm{g}_{\mathrm{v}}(t)=\sqrt{e} \cdot \mathrm{m}_{v}(\mathrm{t})$.

\section{The Resting Conditions}

Full description of the particle populations necessitates the definition of the initial conditions. We suppose that at $\mathrm{t}<0$ the both populations are at the resting state, and use linear BDP [17] as the model of this condition. We define the rates of the death and birth by the following constants:

$\mu_{\pi}=\mu_{v}=1 / \chi, \quad \lambda_{\pi}=\lambda_{v}=1 / \chi$.

\section{GMP Model at Microscopic Scale}

We have expressed $g_{\pi}(t)$ and $g_{v}(t)$ in terms of chaotic BDPs. The basic probabilistic assumption is that during a sufficiently small element of time $\Delta t$ the probability of the change of the population size by more than one element is negligibly small:

$\operatorname{Pr}[\mathbf{X}(t+\Delta t)=\mathbf{X}(t)+m]=o(\Delta t)$ if $|m|>1$,

where Pr denotes probability and $m$ is an integer.

The probabilities of permitted changes in the particle populations are:

$\operatorname{Pr}[\mathbf{X}(t+\Delta t)=\mathbf{X}(t)+1]=\lambda(t) \mathbf{X}(t) \Delta t+o(\Delta t) \quad($ birth $)$

$\operatorname{Pr}[\mathbf{X}(t+\Delta t)=\mathbf{X}(t)-1]=\mu(t) \mathbf{X}(t) \Delta t+o(\Delta t)$

Accordingly, the probability of keeping the population size unchanged is

$\operatorname{Pr}[\mathbf{X}(t+\Delta t)=\mathbf{X}(t)]=1-[\lambda(t)-\mu(t)] \mathbf{X}(t) \Delta t+o(\Delta t)$.

We use these conditions as a basis for numerical investigation of above defined chaotic processes. We deal with the samples $\mathrm{X}_{\pi}(t)$ and $\mathrm{X}_{v}(t)$ of chaotic $\{\lambda, \mu(t), \chi\}$ and $\{\mu(t), \chi\}$ processes, respectively. As an initial condition, we suppose that $\mathrm{X}_{\pi}(0)=\mathrm{X}_{v}(0)$.

The following changes of $\mathrm{X}_{\pi}(t)$ and $\mathrm{X}_{v}(t)$ are computed step by step at the intervals from $t_{\mathrm{i}}$ to $t_{\mathrm{i}+1}$, where $t_{i}=i \Delta$ and $\mathrm{i}$ takes values from 0 to I. Thus we deal with the discrete time, and may regard $X_{i}^{\pi}=\mathrm{X}_{\pi}\left(t_{i}\right)$ and $X_{i}^{v}=\mathrm{X}_{v}\left(t_{i}\right)$ as the states of particle systems. The choice of the value for $\Delta$ is supported by the same notions as $\Delta \mathrm{t}$ in (9)-(11). Therefore, we can express general probabilistic statements (10) and (11) using parameters of concrete chaotic processes.

Given the birth and death rates from (4), it follows rather straightforwardly from (10) and (11) that

$\mathrm{p}_{b}^{\pi}(i)=X_{i}^{\pi} \Delta / \chi, \quad \mathrm{p}_{d}^{\pi}(i)=X_{i}^{\pi} \Delta^{2} i / \chi^{2}$,

where $\mathrm{p}_{b}^{\pi}(i)$ and $\mathrm{p}_{d}^{\pi}(i)$ are the probabilities of a particle birth and death, respectively, in the interval from $t_{\mathrm{i}}$ to $t_{\mathrm{i}+1}$. 
With regard to the death rate from (6),

$\mathrm{p}_{d}^{v}(i)=X_{i}^{v} \Delta(i \Delta+\chi) / \chi^{2}$,

where $\mathrm{p}_{d}^{v}(i)$ is the probability of a particle death at the interval from $t_{\mathrm{i}}$ to $t_{\mathrm{i}+1}$.

The transition from $X_{i}^{\pi}$ to $X_{i+1}^{\pi}$ may have the following outcomes: $X_{i+1}^{\pi}=X_{i}^{\pi}-1, X_{i+1}^{\pi}=X_{i}^{\pi}$ or $X_{i+1}^{\pi}=X_{i}^{\pi}+1$. In the case of the sink, $X_{i+1}^{v}=X_{i}^{v}-1$ or $X_{i+1}^{v}=X_{i}^{v}$. Given the values of probabilities from (12) and (13), the inter-state transitions are calculated using Monte-Carlo simulations of the corresponding birth and death processes.

Numerical experiments were supported by a specially designed computer program written in the object Pascal language of Embarcadero Delphi 2010.
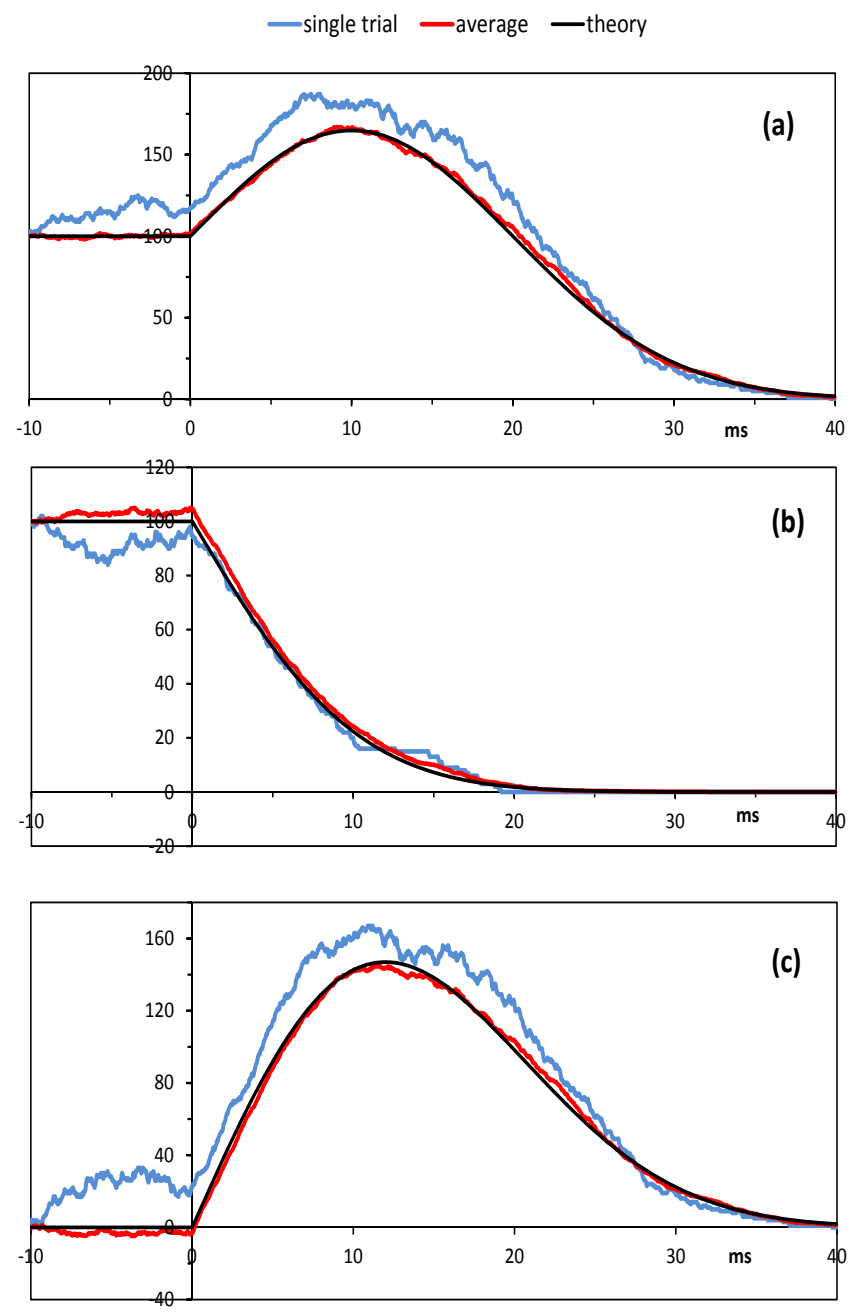

Fig. (3). Illustrates dynamics of particle populations under the resting and transient conditions. The y-axis units are the numbers of particles.

For the sake of illustration the chaos factor was set to be $\chi=10 \mathrm{~ms}$; the value compatible with the time scale of conventional ECG. Typical results of numerical experiments are exemplified by Fig. (3) which compares numerical solutions with theoretical functions $a \cdot \mathrm{g}_{\pi}(t), a \cdot \mathrm{g}_{\mathrm{v}}(t)$, and $a \cdot \mathrm{g}(t)$ where $a$ is a coefficient defined in such a way that $a \cdot \mathrm{g}_{\pi}(0)=a \cdot \mathrm{g}(0)=N_{0}, N_{0}=100$ is the number of particles taken as the initial condition for simulations. The black lines in Fig. (3a-c) are the graphs of the theoretical functions at the interval from -10 to $40 \mathrm{~ms}$. The transients were induced at $\mathrm{t}=0$, and preceded by the resting conditions during which $a \cdot \mathrm{g}_{\pi}(t)=a \cdot \mathrm{g}_{v}(t)=N_{0}(\mathrm{t}<0)$.

The segmentation points for numerical calculations were $t_{i}=i \cdot \Delta$, where $i$ takes values from $-5,000$ to 20,000 and $\Delta=0.002 \mathrm{~ms}$. In the time interval from $-10 \mathrm{~ms}$ to 0 the particle populations in both compartments were simulated as simple BDPs with constant rates of the birth and death (8). As an initial condition it was supposed that the size of each compartments at $\mathrm{t}=-10 \mathrm{~ms}$ is equal to $\mathrm{N}_{0}$. The transition from the resting to transient condition was simulated as the change of the constant rates of the birth and death (8) to the time dependent rates (4) and (6) of chaotic $\{\lambda, \mu(t), \chi\}$ and $\{\mu(t), \chi\}$, respectively. The blue lines in (a) and (b) show $\mathrm{X}_{\pi}(t)$ and $\mathrm{X}_{v}(t)$ from one and the same trial. The blue line in (c) is $\mathrm{X}(t)=\mathrm{X}_{\pi}(t)-\mathrm{X}_{v}(t)$.

To define the limits to which the statistical solutions converge, the averages of single trial samples were computed. The red lines in (a)-(c) show the averages of ten samples obtained in independent trials. This example, as well as the results of similar computations with different parameter sets, unambiguously indicates the consistency of statistical outcomes with expected theoretical dependencies.

A key role in the dynamics of $g_{\pi}(t)$ and $g_{v}(t)$ can be associated with the specific combination of random and deterministic factors that produce a deterministic chaos. An important aspect of chaotic dynamics is a nonlinearity of underlying system. Note that the variable which describes the state of the system in (12) and (13) is the size of the particle population, i.e $X_{i}^{\pi}$ or $X_{i}^{v}$. Multiplication of these variables by $t_{i}=i \Delta$ is the source of nonlinearities at microscopic scale.

\section{The Chaotic Transformations Effect}

The role of deterministic factors in statistical samples increases with increase in the number of particles involved. Single trial samples shown in Fig. (3) develop from initial populations of 100 particles. A tenfold increase of $\mathrm{N}_{0}$ to 1000 particles significantly reduces variability and brings $\mathrm{X}_{\pi}(t)$ and $\mathrm{X}_{v}(t)$ to a better proximity with deterministic solutions. On purely theoretical grounds we may regard the Gaussian components of standard GMP (2) as the limits to which the transients converge with an infinite increase of the population size.

However, the situation is not that simple when we regard the chaotic BDP as a physical model. The point is that calculations of statistical samples should be consistent with condition (9). Therefore, the probabilities of the birth and death of a particle during the $\Delta$ interval should be low. Given the experimental runs from Fig. (3), the values of all 
probabilities from (12) and (13) were usually (98\% of trials) below 0.065 . Specially designed tests with different types of BDPs, as well as the previous experience in simulations of transmembrane particle transfers [15], indicate that this range of probabilities provides correct interpretation of the condition (9) in numerical simulations.

A free parameter that allows us to keep the values of probabilities at low levels is the sampling interval $\Delta$. Physically, the value of this parameter should be compatible with the time interval that is necessary for an ion channel to change the state; this event moves an ion through the membrane. With regard to the millisecond order of such transition, the value $\Delta=0.002 \mathrm{~ms}$ used in above simulations may be considered as a reasonable parameter. However, the corresponding size of the particle population (100-200 units) is small compared with actual numbers of ions participating in the trans-membrane transport processes. Even in the case of a single cell, the number of excitable channels is estimated to be large (on the order of tens of thousands of ion channels). To keep the birth and death probabilities at reasonably low levels in the case of such large particle populations, it is necessary to reduce sampling intervals to physically unrealistic values.

To avoid such situation and resolve the problem in a physically acceptable way, we refer to the independence principle according to which the behavior of a particular group of ions involved in the transmembrane transport processes is independent of the number and type of other participating ions [18]. Using this principle, we divide the whole particle population into the sub-populations each of which is described by chaotic $\left\{\lambda, \mu(t), \chi^{k}\right\}$ and $\left\{\mu(t), \chi^{k}\right\}$ processes specified by the chaos factors $\chi^{k}(k$ is an index of particular population). Given equal initial sizes and $\chi^{k}$ values of the $\chi$-factor for each sub-population, we actually deal with the same realizations of the chaotic process as those reproduced in numerical experiments. Indeed, note that the red lines in Fig. (3a, b) are the averages of 10 independent samples of chaotic processes produced by a single particle population. We may as well interpret these results as the averages of single trial samples produced by 10 independent populations with equal parameters.

However, the sub-populations are the models of different groups of ions associated with a single cell or a cell ensemble. Therefore, it is reasonable to propose that different sub-systems have different parameters. We analyzed this situation in numerical experiments. Typical results illustrated by Fig. (4) deal with the model composed of $50 \mathrm{sub}$-systems with different parameters. The minimum and maximum values of parameters were 7 and $12 \mathrm{~ms}$ (mean $9.89 \mathrm{~ms}$ ) for the $\chi$-factor, and 76 and 125 (mean 101) for initial size of the population at $\mathrm{t}=0$. The parameter distributions between these extremes, are illustrated by the histograms in (a) and (b).

Fig. (4c) refers to the function $X^{k}(t)=X_{\pi}^{k}(t)-X_{v}^{k}(t)$, where $X_{\pi}^{k}(t)$ and $X_{v}^{k}(t)$ are the samples of chaotic processes $\left\{\lambda, \mu(t), \chi^{k}\right\}$ and $\left\{\mu(t), \chi^{k}\right\}, \quad$ respectively.

Given the parameter values indicated by the histograms, each of the 50 systems under the analysis is presented by a trace of $X(t)$. The red solid line is the average of these traces. The black dotted line is the theoretical solution for particle population with $\bar{N}_{0}$ and $\bar{\chi}$ parameters. These are the mean values of $N_{0}^{k}$ and $\chi^{k}$ parameters; $\bar{N}_{0}=101, \quad \bar{\chi}=9.89 \mathrm{~ms}$.
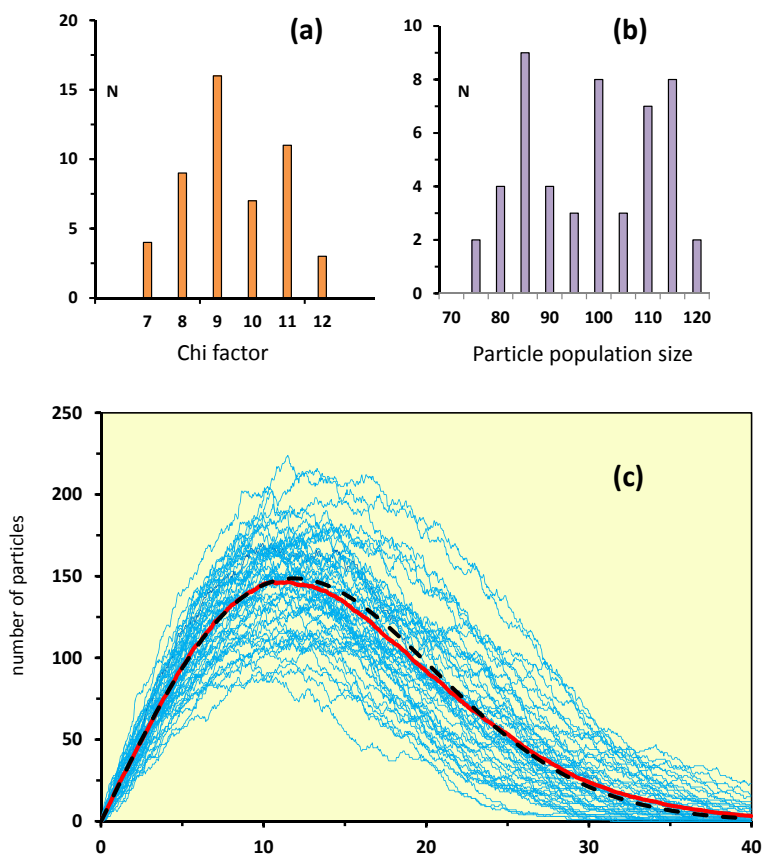

Fig. (4). Illustrates the chaotic transformations effect.

This and similar numerical experiments show that differences in the sizes of particle populations and $\chi$ factors do not affect the general form of statistical solutions (5) and (7). No matter how complex the particle system, from the viewpoint of a chaotic dynamics, the system behaves as if it consisted only of a single dipole. The parameters of this equivalent dipole, $\bar{N}_{0}$ and $\bar{\chi}$, represent the mean values of the corresponding parameters of participating subpopulations. We qualify this finding as the chaotic transformations effect.

\section{Nonlinear Equations of GMP (Global Scale)}

We present an explicit description of the system producing deterministic chaos in the form of the following system of nonlinear differential equations:

$$
\begin{aligned}
& \dot{\mathrm{x}}(\mathrm{t})=-\frac{t-\chi}{\chi^{2}} \mathrm{x}(t), \\
& \dot{\mathrm{y}}(\mathrm{t})=-\frac{t+\chi}{\chi^{2}} \mathrm{y}(t), \\
& \mathrm{z}(t)=\frac{\mathrm{x}(t)-\mathrm{y}(t)}{\sqrt{2 \pi}} \cdot 1(\mathrm{t}),
\end{aligned}
$$

where $\dot{\mathrm{x}}(t)$ and $\dot{\mathrm{y}}(t)$ denote the derivatives of the state variables $\mathrm{x}(t)$ and $\mathrm{y}(t)$ with respect to the time $\mathrm{t}$. 
Since the state variables in (14) and (15) are multiplied by $t$, the system is non-autonomous, i.e. it is driven by timevarying signals. Substitution of $g_{\pi}(t)$ and $g_{v}(t)$ into (14) and (15), respectively, shows that these functions are the solutions of the system. The initial conditions are settled by the unit step function in (16).

The form of (14)-(16) is consistent with the notion that equations of deterministic chaos must be non-linear to generate chaotic solutions, but apart from that, they can be remarkably simple. Prior to these results, the analytical models of chaotic systems in the field of the heart research were very few. Thus, such active area for applications of the chaos theory as the heart rate variability is not supported by the basic nonlinear equations [19].

The generalization of our global scale model in the form of nonlinear equations provides access to various methods of nonlinear dynamical analysis, such as the Poincare map, the frequency domain transfer function, attractors, Lyapunov exponents, etc. We use in this study the frequency domain methods to construct a tool for identification of specific "chaotic" elements of ECG waveforms.

\section{The Frequency Domain}

Given the standard GMP (2) as a function of time, we regard bud(t) as an impulse response of the system described by (14)-(16), i.e. the transient induced by a Dirac delta impulse. This allows us to define the frequency transfer function of the system by a classical one sided Fourier integral

$\mathrm{B}(\omega)=\mathrm{B}_{R}(\omega)-i \mathrm{~B}_{I}(\omega)=\int_{0}^{\infty} \operatorname{bud}(t) e^{-i \omega t} d t$,

where $i=\sqrt{-1}, \omega=2 \pi f$, and $\mathrm{f}$ is frequency. The imaginary part function is expressed analytically in the form

$\mathrm{B}_{I}(\omega)=\exp \left(-\omega^{2}\right) \sin \omega$.

We were unable to find an analytical solution for the real part function $\mathrm{B}_{R}(\omega)$, and constructed instead a reasonably accurate approximant in the form $\mathrm{B}_{R}(\omega) \approx \mathrm{R}(\omega)=$ $\mathrm{R}_{1}(\omega)+\mathrm{R}_{2}(\omega)$, where

$\mathrm{R}_{1}(\omega)=\exp \left(-\omega^{2}\right) \cos \omega$

and $\mathrm{R}_{2}(\omega)=-1.204 \cdot \exp \left(-\omega^{2} / 1.518\right)$.

On this basis,

$\mathrm{B}^{M}(\omega)=|\mathrm{B}(\omega)| \approx \sqrt{\mathrm{B}_{R}^{2}(\alpha)+\mathrm{B}_{I}^{2}(\omega)}$.

For the range of $\alpha$ from $\omega=0$ to $\omega=2.5$

$\mathrm{B}^{M}(\omega) \approx \frac{2}{s} \mathrm{e}^{-\omega^{2} / 2 s^{2}}$,

where $\mathrm{s}=1.364$.

We will use this function as a frequency domain template for the identification procedure described in the section (3.5).

\section{IDENTIFICATION OF THE CHAOS}

\section{Conceptual Framework}

To apply our theory to the ECG analysis, we now consider how to reconcile the ECG waveform with the dynamics of chaotic processes. Almost without exception, current approaches to the ECG analysis regard "peak" synonymous to a functional component. It is widely accepted to consider the ECG as a set of waves, to pick the peaks of these waves, and to measure the peak amplitude and latency. On an understanding of a component as being not just a peak in the waveform, but a whole deflection with particular shape, we assume that underlying system at the microscopic scale can be adequately described in terms of chaotic BDPs. The conceptual framework is that rather than a continuous process, the ECG represents a series of consecutive, partly overlapping transient potentials, each of which is produced through intermittent synchronization of specific ensemble of the heart muscle cells in a local conductance volume. Chaotic dynamics of these processes combines deterministic and random components which coexist at the microscopic scale level. However, the random components are hidden at the global level because the mass effect of large particle populations emphasizes coherent components and supresses random components. Therefore, we may associate the dynamics of ECG waveforms with the mean values (5) and (7) of chaotic BDPs, i.e. with GMPs.

\section{Fragmentary Decomposition}

The question is how to identify these GMPs on empirical basis? Based on previous work, we implement the method of high resolution fragmentary decomposition [12]. Taking the ECG to be $v(t)$, we divide $v(t)$ into the segments by the points $\tau_{\mathrm{i}}(i=0, . ., . N)$ in which $\mathrm{v}(\mathrm{t})$ has zero crossing or $|\mathrm{v}(\mathrm{t})|$ has a minimum.

The piece of $v(t)$ between adjacent segmentation points is termed a half-wave function (HWF), and defined as

$\mathrm{w}_{\mathrm{k}}(\mathrm{t})=\left\{\begin{array}{c}\mathrm{v}\left(\mathrm{t}+\tau_{\mathrm{k}-1}\right) \text { if } 0 \leq \mathrm{t} \leq \lambda_{\mathrm{k}}, \\ 0 \text { otherwise }\end{array}\right.$

where $\lambda_{\mathrm{k}}=\tau_{\mathrm{k}}-\tau_{\mathrm{k}-1}$.

In terms of HWFs, the ECG is

$\mathrm{v}(t)=\sum_{k=1}^{N} \mathrm{w}_{k}\left(t-\tau_{k-1}\right)$.

Phenomenologically, $\mathrm{w}_{\mathrm{k}}(\mathrm{t})$ may be associated with development of the $k$ th peak of $v(t)$. On assumption that generation of a peak is produced by chaotic processes described by our theory, we may regard GMP as an adequate model of the HWF. Therefore, we may present ECG by the following fragmentary model

$\mathrm{u}(t)=\sum_{k=1}^{N} \mathrm{~g}_{k}(t)$

where 
$\mathrm{g}_{\mathrm{k}}(t)=\kappa_{k} \cdot$ bud $\left[\left(t-\psi_{k}\right) / \lambda_{k}\right]$.

A criterion that establishes relationships between the model (19) and empirical ECG (18) is the interpolation condition

$\mathrm{u}\left(L_{k}\right)=\mathrm{v}\left(L_{k}\right) \quad(k=1, \ldots, P)$,

where $\mathrm{L}_{\mathrm{k}}$ is the peak latency of the $k$ th peak (interval from $\tau_{\mathrm{k}-1}$ to $\tau_{k}$ ). Consequently, the peak latencies and amplitudes of the model must be equal to the peak latencies and amplitudes of the original ECG.

\section{Resolution of the Component Overlap}

The possibility of a component overlap is an important concern with regard to the identification process. After [12], we implement a model based approach to the problem.

Let us regard the $m(m<N)$ first terms from (19),

$\mathrm{u}_{m}(t)=\sum_{k=1}^{m} \mathrm{~g}_{k}(t)$,

as the solution of identification problem for the ECG segment from $\tau_{0}$ to $\tau_{m}$. By this we particularly mean that $\mathrm{u}_{\mathrm{m}}(\mathrm{t})$ captures the ECG dynamics in the interval from $\tau_{\mathrm{m}-1}$ to $\tau_{\mathrm{m}}$. The essence of the model based approach is that we use an analytical $\mathrm{u}_{\mathrm{m}}(\mathrm{t})$ as an extrapolation tool which enables us to predict the development of the ECG waveform at $\mathrm{t}>\tau_{\mathrm{m}}$. For the following $\left[\tau_{m}, \tau_{\mathrm{m}+1}\right]$ segment, we define an overlap corrected HWF in the form

$\tilde{\mathrm{w}}_{m+1}(t)=\mathrm{v}\left(t+\tau_{m}\right)-\mathrm{u}_{m}\left(t+\tau_{m}\right) . \quad\left(0 \leq t \leq \lambda_{m}\right)$

This formula supports processing of the kth segment by the parameters of the previous segment (for the first segment $\left.\mathrm{g}_{0}(\mathrm{t})=0\right)$. Using this opportunity, the identification of GMP parameters is performed in successive steps from the first to the last segments of the signal. At each step our aim is to estimate the values of GMP parameters which provide the best fit to the corresponding overlap corrected HWF. To estimate the parameters using the minimization routines which are generally employed in this context, we need to organize an iterative search of an optimal solution in a 3dimensional parameter space. We implement more straightforward approach using the frequency domain identification technique.

\section{Time-Frequency Analysis}

The idea is to use a characteristic Gaussian profile (17) of GMP amplitude spectrum as a frequency domain template for comparison with the amplitude spectrum of HWF $\mathrm{W}_{k}^{M}(\omega)=\left|\mathrm{W}_{\mathrm{k}}(\omega)\right|$, where $\mathrm{W}_{\mathrm{k}}(\omega)$ is the complex spectrum defined by the following finite Fourier integral

$\mathrm{W}_{\mathrm{k}}(\omega)=\int_{0}^{\lambda_{\mathrm{k}}} \mathrm{W}_{\mathrm{k}}(\mathrm{t}) \cdot \mathrm{e}^{-\mathrm{it} \omega} \mathrm{dt}$.

This standard form of short-time Fourier transform demands special algorithms of numerical integration. We use the similar basis function (SBF) algorithm which is adequate to the time-frequency analysis, i.e. the spectral analysis of successive signal segments of arbitrary length [20,21].

We specify $w_{k}(t)$ by its sampled values $w_{k}\left(t_{i}\right)$ in a finite number of nodal points $t_{i}(i=0,1, . ., N)$ with $t_{0}=0$ and $t_{N}=\lambda_{k}$. The nodes need not be spaced equally. The algorithm creates a piece-wise linear interpolant and decomposes it into the sum of finite elements termed "similar basis functions". The algorithm provides means for an explicit treatment of discontinuities at the boundaries of the integration interval. This eliminates the need for windows for spectral analysis, along with their distorting impact.

As a means to control the accuracy of the calculations, we restore the initial signal from the real or imaginary part of the complex spectrum $\mathrm{W}_{\mathrm{k}}(\omega)$ using the SBF algorithm for calculation of relevant inverse Fourier transform.

\section{Frequency Domain Template Matching}

An apparent advantage of the frequency domain is that amplitude spectrum $\mathrm{W}_{k}^{M}(\omega)$ is invariant to the time shifts of the HWF. This removes the parameter $\psi$ of the corresponding GMP from the frequency domain identification procedures.

The frequency domain procedures deal with the normalized functions

$\overline{\mathrm{B}}^{M}(\omega)=\mathrm{B}^{M}(\omega) / \mathrm{B}^{M}(0)$ and $\quad \overline{\mathrm{W}}^{M}(\omega)=\mathrm{W}^{M}(\omega) / \mathrm{W}^{M}(0)$. Fig. (5b) illustrates $\overline{\mathrm{B}}^{M}(2 \pi f)$ for the following values of $\chi$ : dash-dot line $-30 \mathrm{~ms}$, solid line $-11 \mathrm{~ms}$ and dash line -3 ms. The change of $\chi$ does not alter the form of $\overline{\mathrm{B}}^{M}(2 \pi f)$ but shifts the entire function along the frequency axes. Thus, it is a simple matter to find a best fit of theoretical $\overline{\mathrm{B}}^{M}(2 \pi f)$ to empirical $\overline{\mathrm{W}}_{k}^{M}(2 \pi \mathrm{f})$

which gives the estimate of $\chi_{k}$.

The criterion that establishes the estimates of remaining parameters is the interpolation condition (20). Given that standard GMP (2) has its maximum at $\mathrm{x} \approx 1.2$, it is easily deduced from this condition that $\psi_{\mathrm{k}}=\mathrm{L}_{\mathrm{k}}-1.2 \chi_{\mathrm{k}}$. The corresponding amplitude value is $\kappa_{\mathrm{k}}=\tilde{\mathrm{w}}_{k}\left(1.2 \chi_{k}\right) / \operatorname{bud}(1.2)$.

\section{Self-Similar Waveforms and Standard Variables}

Suppose that $g_{k}(t)$ is an identified model of $w_{k}(t)$. How can we compare these geometrical forms and test the validity of the GMP as a model or improve the parameter estimates? We approach this problem using the self-similarity of GMPs. Given a pair of GMPs with different parameters, it follows from (1) and (2) that

$$
\frac{1}{\kappa_{i}} \mathrm{~g}_{i}\left(\chi_{i} x+\psi_{i}\right)=\frac{1}{\kappa_{j}} \mathrm{~g}_{j}\left(\chi_{j} x+\psi_{j}\right)=\operatorname{bud}(x) \text {, }
$$

where $\mathrm{i}$ and $\mathrm{j}$ can take values from 0 to $\mathrm{N}, \mathrm{i} \neq \mathrm{j}$. 

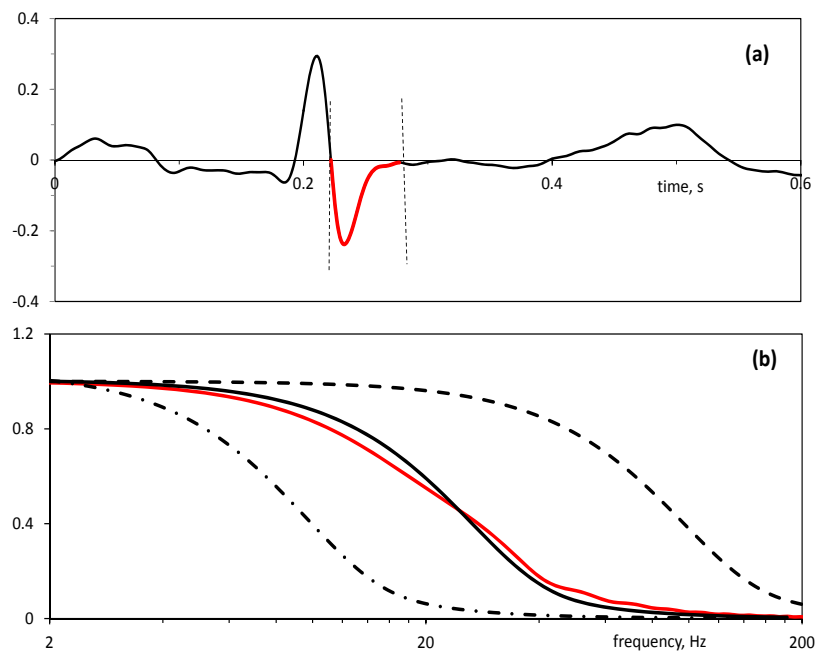

Fig. (5). Identification of the $\chi$ factor using the time-frequency analysis. (a) Shows the ECG (black line) and the HWF (red line) selected for identification. The y-axis units are mVs. (b) The red line shows the amplitude frequency spectrum of the HWF from (a). The black lines show different positions of the frequency domain template. The y-axis displays dimensionless units.

This formula transfers GMP from the space of physical variables (voltage as a function of time) to the space of dimensionless or standard variables (dimensionless amplitude as a function of standard variable $\mathrm{x}$ ) where the GMP degenerates into the dimensionless waveform of standard GMP. Therefore, the GMPs may be regarded as an ensemble of self-similar functions the waveforms of which are identical in the space of standard variables.

Given the parameter set, $\kappa_{\mathrm{k}}, \psi_{\mathrm{k}}$ and $\chi_{k}$, the transformation of corresponding overlap corrected HWF has the form

$$
\mathrm{z}_{k}(x)=\frac{1}{\kappa_{k}} \mathrm{w}_{k}\left(\chi_{k} x+\psi_{k}\right) \text {. }
$$

The power of this normalization is that it removes diversity in the physical measures of initial empirical waveforms and provides dimensionless bud(x) as a golden standard for the analysis of modelling accuracy. The residual, $\mathrm{r}_{k}(x)=\mathrm{z}_{k}(x)-\operatorname{bud}(x)$, is a universal measure of the HWF deviation from the model in the space of standard variables.

\section{Software}

The computer implementation of the identification procedures has been supported by specially designed software package "FD_ECG" using the object Pascal language of Embarcadero Delphi 2010. The functional organization of the FD_ECG includes the three major processing engines.

First, an automated analysis engine the major processing routines of which perform the following operations: (1) ECG artifact elimination using digital recursive boxcar filters, (2) ECG adaptive segmentation and selection of HWFs, (3) short-time spectral analysis of HWFs, (4) identification of
GMP parameters, (5) control of modelling accuracy using inverse Fourier transforms and standard variables.

Second, the visualization engine designed as a tool for visualizing the analysis results. This tool provides means to display the results of automated analysis for any specified ECG segment. The image includes the original ECG, the waveforms of identified GMPs and the values of estimated parameters.

Third, the engine of the time-frequency analysis using the SBF algorithm. This analysis and visualization tool provides means to analyse selected ECG segments and compare their spectral profiles with standard frequency characteristics of typical elements of control systems.

\section{RESULTS}

\section{ECG Recording and Processing}

The ECG data analysed for this paper came from two independent sources.

First, ambulatory ECGs were recorded over ten seconds from patients in an outpatient cardiac clinic. Standard 12lead surface electrocardiograms were obtained in the recumbent resting position with a microprocessor-based commercial system (Mortara Instruments Inc.) at a sampling rate of $1000 \mathrm{~Hz}$. Each acquisition was obtained in triplicate, with the last or most visually artifact-free trace used for further quantitative analysis. ECGs of 20 subjects without heart disease were selected for this study.

Second, set of ECG recordings of normal subjects were obtained from the PTB diagnostic ECG database [22, 23]. Being available over the Internet, the PTB database is the source of high fidelity digital ECG records. Each recording has duration of $115.2 \mathrm{sec}$ and includes 15 simultaneously measured signals at sampling frequency of $1 \mathrm{kHz}$ : the conventional 12 leads together with the 3 Frank lead ECGs. For this study we have selected ECGs of 20 normal subjects. For each of these subjects, the ECG data for analysis were prepared in the same format as for the first group of subjects, i.e. 10,001 point time series $(10 \mathrm{~s}$ segment from the beginning of the record) from each of 12 conventional leads.

The automated analysis of ECGs from these 40 subjects was performed on 12-channel, 10-sec ECG segments running the FD_ECG automated analysis routine on a Compaq Presario $3.06 \mathrm{GHz}$ PC with $4 \mathrm{~GB}$ RAM. The analysis results have been examined using the visualization and time-frequency analysis engines. To support our theory by empirical data, we describe below some typical results which were observed in the both ECG sources.

\section{Major ECG Waveforms}

The solid black line in Fig. (6) is a 601 point time series (1 ms sampling interval) which shows a typical ECG during a heartbeat. The colored lines are model waveforms superimposed on the corresponding $\mathrm{P}, \mathrm{Q}, \mathrm{R}, \mathrm{S}$ and $\mathrm{T}$ components.

This gives a visual impression of reasonable accuracy with which the equations of chaotic BDPs describe the 
dynamics of a real ECG. The parameters of identified BPDs are shown in the Table $\mathbf{1 .}$

The values of the chaos factor are also displayed by the bars in the right upper panel of Fig. (6).

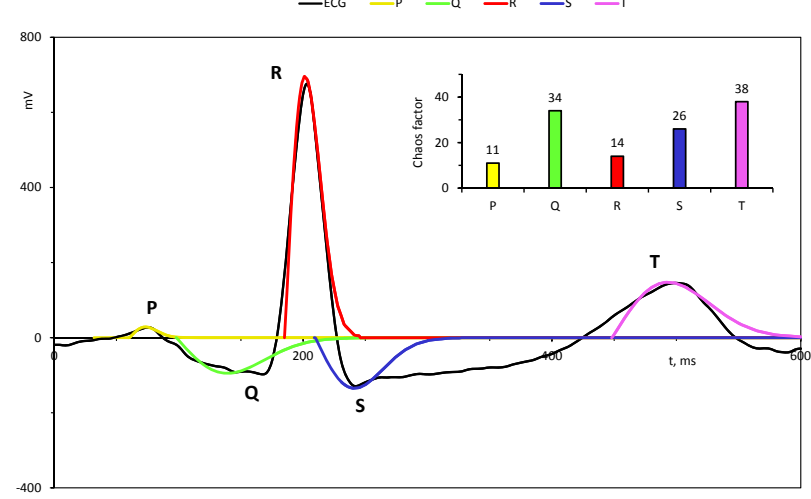

Fig. (6). Model GMPs are superimposed on the major P, Q, R, S and $\mathrm{T}$ components.

Table 1. Parameter Values of Identified GMPs from Fig. (6)

\begin{tabular}{|c|c|c|c|c|c|}
\hline & $\mathbf{P}$ & $\mathbf{Q}$ & $\mathbf{R}$ & $\mathbf{S}$ & $\mathbf{T}$ \\
\hline \hline$\kappa, \mu \mathrm{V}$ & 80.5 & -268 & 1960 & -380 & 416 \\
\hline$\Psi, \mathrm{ms}$ & 73 & 98 & 185 & 210 & 448 \\
\hline$\chi, \mathrm{ms}$ & 11 & 34 & 14 & 26 & 38 \\
\hline
\end{tabular}

An equally satisfactory description of particular ECG waveform could no doubt have been achieved with equations of very different form. However, different models may yield similar results, but one may require fewer model parameters and is therefore more efficient in handling the physics of the process. In this regard, a remarkable aspect of nonlinear equations (14)-(16) is that the solution in the form of GMP contains a minimum number of free parameters, $\kappa, \psi$ and $\chi$. $\kappa$ and $\psi$ are similar to conventional measures of the amplitude and timing of the ECG waveform. Hence, just a single parameter, the $\chi$ factor, appears as an inclusive measure of the waveform dynamics without any adjustment to make the nonlinear equations fit the features of particular waveform.

The success of the theory in predicting the dynamics of ECG waveforms encouraged us to examine in a more detail a complex component composition of ECG waveforms. Historically, each of conventional P, Q, R, S and T components of ECG has been associated with specific cellular process. This outlook has shaped virtually every aspect of the ECG waveform analysis, particularly the concept of a monolithic component, and the lack of attention to the component temporal overlap. Our results reveal considerably more complex and informative dynamics of conventional ECG waveforms than previously thought. Below we address this issue to the morphology of $\mathrm{Q}$ and $\mathrm{S}$ waves.

\section{Composite Structure of Q and S Waves}

The QRS complex is the most characteristic waveform of the ECG signal. The high amplitude, positively deflecting R wave makes detection of the QRS complex easier than the other waves. Thus, the $\mathrm{R}$ wave is generally used as a reference within the cardiac cycle. Once the $\mathrm{R}$ wave is identified, the $\mathrm{Q}$ and $\mathrm{S}$ waves are conventionally regarded as negative ECG deflections before and after R, respectively. Actually, the shapes of such defined morphological elements of the ECG show remarkable diversity of geometrical forms. In some cases the technique of high resolution FD resolves the $Q$ and/or $S$ waves into the sum of slow and fast components.

Fig. (7a) shows a characteristic example of ECG with complex negative deflection preceding the $\mathrm{R}$ wave. The FD decomposes this waveform into the earlier slow wave $\mathrm{SQ}$ with $\chi=32 \mathrm{~ms}$ and the later fast wave fQ with $\chi=10$.

A composite $\mathrm{S}$ wave is exemplified by Fig. (7b). The results of FD indicate that negative ECG deflection following the $\mathrm{R}$ wave consists from the earlier fast fS component with $\chi=8 \mathrm{~ms}$ and the later slow component $\mathrm{sS}$ with $\chi=15 \mathrm{~ms}$.

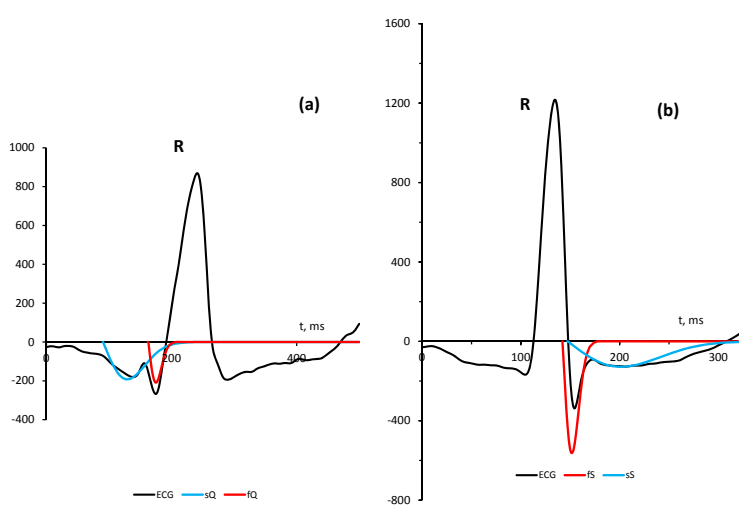

Fig. (7). Examples of complex $\mathrm{Q}$ and $\mathrm{S}$ waves. (a) Illustrates the slow Q (sQ) which develops before conventional Q wave. (b) The $\mathrm{R}$ wave is followed by an almost simultaneous induction of the conventional S wave and slow S (sS). The y-axis units in (a) and (b) are $\mu \mathrm{Vs}$.

The morphology of identified components allows us to propose that $\mathrm{fQ}$ and $\mathrm{fS}$ are the conventional $\mathrm{Q}$ and $\mathrm{S}$ waves, i.e. the elements of the QRS complex. By contrast, striking differences in the values of the $\chi$ factor of slow and fast components indicate a distinct nature of slow components.

\section{Double Peak R Wave}

The $\mathrm{R}$ wave is conventionally regarded as a monolithic waveform. However, in some cases the R wave may have a complex structure. In our analysis the complex morphology of the $\mathrm{R}$ wave has been identified in two normal subjects from different groups. In both cases a major peak of the $\mathrm{R}$ wave is followed by a small positive deflection $\mathrm{IR}$ (late $\mathrm{R}$ ) as illustrated in Fig. (8a, the ECG is from the first group). 

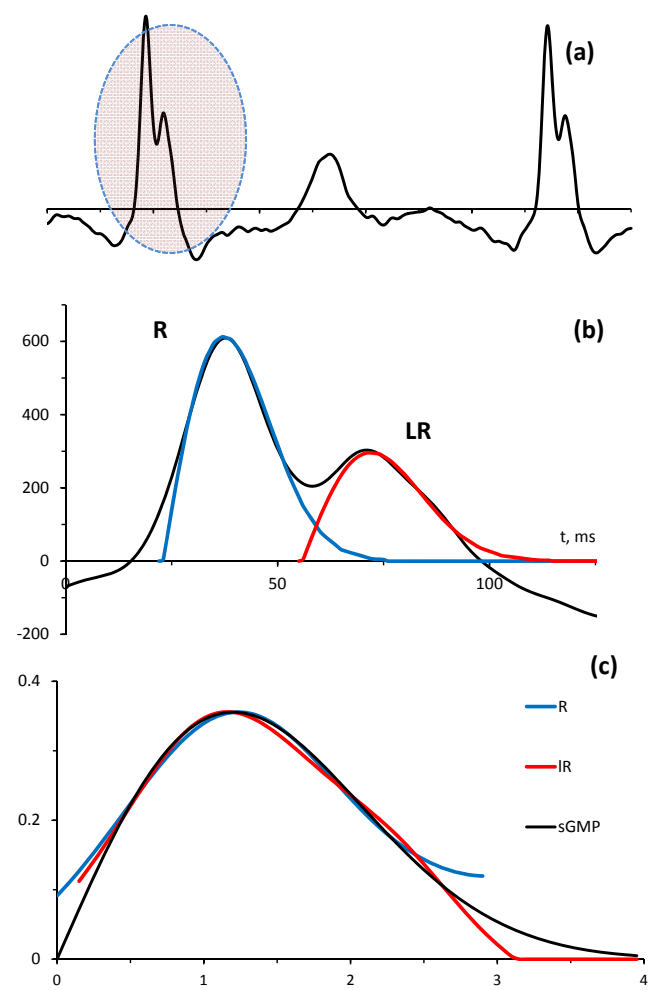

Fig. (8). Example of complex $\mathrm{R}$ wave. (a) ECG segment with complex $\mathrm{R}$ waves. (b) The $\mathrm{R}$ wave highlighted in (a) is decomposed into the two components: conventional $\mathrm{R}$ and late $\mathrm{R}$ (IR). The $y$-axis units are $\mu$ Vs. (c) After transformation to the space of standard variables, the empirical counterparts of $\mathrm{R}$ and $\mathrm{IR}$, i.e. the corresponding HWFs, are compared with the standard GMP (sGMP). The y-axis displays dimensionless units.

Such small waveform perturbations are difficult to study by quantitative analysis using conventional routines. However, it appears that our technique of the component overlap resolution is capable of reliable separation of $1 \mathrm{R}$ from the $\mathrm{R}$ wave. Identified as separate entities, the $\mathrm{R}$ and $\mathrm{IR}$ waveforms are shown in Fig. (8b) in comparison with the corresponding segment of original ECG. The values of the $\chi$ factor for $\mathrm{R}$ and $\mathrm{R}$ are 12 and $13 \mathrm{~ms}$, respectively. The component normalization according to the formula (21) provided a capability for direct comparison of $\mathrm{R}$ and $\mathrm{IR}$ waveforms with the standard GMP in the space of standard variables. A remarkably accurate match of both curves to the template is illustrated by Fig. (8c). Therefore, the dynamics of both components is consistent with nonlinear equations (14)-(16).

Appearance of the late peak in time course of the $\mathrm{R}$ wave is an infrequent event, i.e. the marker of very specific aspects of the heart performance. The relevant conditions in this regard are the hypothermia and high blood levels of calcium [24].

There are clearly several factors at work which complicate recognition and identification of different components of ECG waveforms. A most difficult problem is the resolution of the component temporal overlap. Success of the FD algorithm in a number of practical cases provides basis for further insight into the underlying constituents of the ECG dynamic performance.

\section{Collective Properties of R-S Waves}

According to the conceptual framework of our modeling approach, the ECG signal develops as a series of consecutive, partly overlapping GMPs. Such phenomenological picture should include some form of cooperative actions between underlying cellular systems. Our previous study of the heart self-regulation using the frequency domain identification technique has found that temporal dynamics of QRS complex may be described with reasonable accuracy in terms of classical underdamped harmonic oscillator [25]. These results indicate a highly coordinated temporal dynamic of $\mathrm{R}$ and $\mathrm{S}$ waveforms. To illustrate this type of a global scale dynamics, we take the ECG from Fig. (6) as an example. We describe coordinated activity of the sources of $\mathrm{R}$ and $\mathrm{S}$ components as a transient process $\mathrm{f}(t-185)=\mathrm{g}_{R}(t)+\mathrm{g}_{S}(t)$, where the GMPs are the models of $\mathrm{R}$ and $\mathrm{S}$, respectively. The parameters of these GMPs were taken from Table $\mathbf{1}$ for $\mathrm{R}$ and $\mathrm{S}$ waves. Due to 185 ms time delay, $f(t)$ begins from $t=0$ as illustrated by Fig. (9a). Despite the fact that $g_{S}(t)$ begins $25 \mathrm{~ms}$ after $g_{R}(t)$, the transition is smooth. This gives a visual impression of $\mathrm{f}(\mathrm{t})$ as a continuous transient process.

The frequency domain identification of $f(t)$ using the harmonic oscillator as a model gave the following parameters: damping ratio $\zeta=0.53$, natural frequency $\mathrm{f}_{0}=11.5$ Hz. Fig. (9) illustrates reasonable accuracy with which the characteristics of the oscillator (red lines) fit the empirical dependencies (black lines). What is of particular interest for the understanding the effect of the cooperative action of $\mathrm{R}$ and $\mathrm{S}$ is that the slope of the Bode diagram (frequencies from 10 to $100 \mathrm{~Hz}$ ) is $40 \mathrm{~dB}$ per decade. This unambiguously indicates that the dynamic system is described by a second order differential equation.

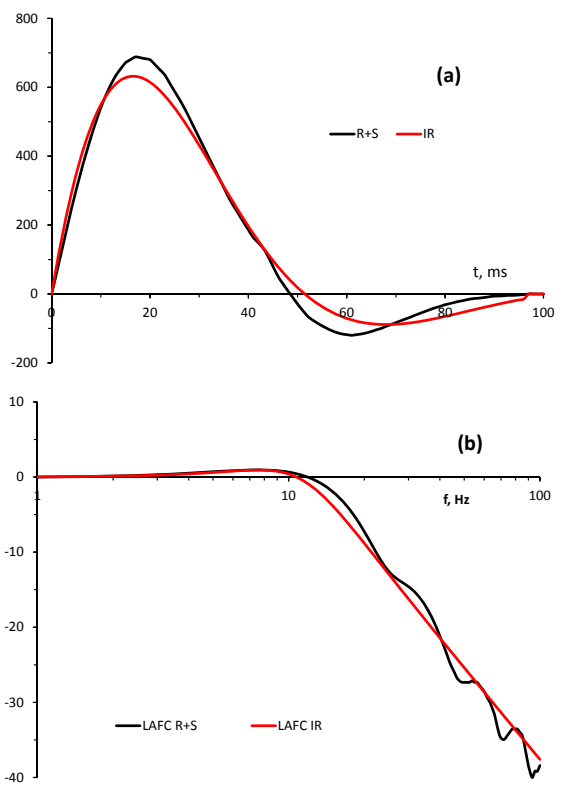

Fig. (9). Transient dynamics of $\mathrm{R}$ and $\mathrm{S}$ waves. (a) Compares the transient process formed as the sum of GMP models of $\mathrm{R}$ and $\mathrm{S}$ waves $(\mathrm{R}+\mathrm{S})$ with the impulse response (IR) of the underdamped harmonic oscillator. (b) Shows the frequency domain counterparts of the transients from (a) in the form of logarithmic amplitude frequency characteristics (LAFC). 
The details of the numerical algorithms and relevant equations can be found in our previously published articles $[21,25]$.

\section{DISCUSSION}

As we demonstrate in this study, a wide range of monolithic deflections identified in the time course of normal ECG may be qualified as self-similar processes that resemble a geometric form of a standard GMP. Rather than a continuous process, the ECG develops as a series of consecutive, partly overlapping self-similar potentials, each of which is produced through intermittent synchronization of specific ensemble of the heart muscle cells in a local conductance volume. We found that dynamical behaviour of GMP ensembles may progress in a highly coordinated fashion. Thus, while the onset of the $\mathrm{R}$ wave leaves behind the onset of the $\mathrm{S}$ wave, the collective effect of both processes is amalgamated on the global level into a single oscillation.

Our theory suggests that fairly simple analytical form of self-similar GMP composed from two shifted Gaussian distributions is rooted in the physics of the mass potentials. And so provides the deterministic chaos as a clue to bring together the deterministic and stochastic factors underlying ECG genesis.

Previous models of ECG emergence from cellular sources have been supported by the landmark HodgkinHuxley $(\mathrm{HH})$ model of conduction and excitation in the cell membrane [2]. However, the applications of HH equations for modelling the cardiac muscle cells are not supported by a unique solution for at least the two following reasons.

The first is a significant redundancy of model parameters. Original HH model consists of the set of four coupled, highly nonlinear ordinary differential equations with multiple parameters [18]. Numerous modifications of $\mathrm{HH}$ model provide more condensed descriptions of particular phenomena. For example, an elegant two-parameter model of FitzHugh [26] allowed the rigorous analysis of action potentials in terms of the fast action potential and a slow recovery variable.

The second is a gap between purely deterministic equations of $\mathrm{HH}$ model and probabilistic notions that support the central kinetic relationships. The key objects in this context are the voltage dependent conductances $\mathrm{g}_{\mathrm{K}} \propto \mathrm{n}^{4}$ and $\mathrm{g}_{\mathrm{Na}} \propto \mathrm{m}^{3} \mathrm{~h}$ for $\mathrm{K}^{+}$and $\mathrm{Na}^{+}$channels, respectively. Hodgkin and Huxley [18] note that the type of kinetics described by $\mathrm{g}_{\mathrm{K}}$ is obtained on assumptions that there are four identical particles, each with probability $n$ of being in the correct position to have an open $\mathrm{K}^{+}$channel. The term $\mathrm{m}^{3} \mathrm{~h}$ in $\mathrm{g}_{\mathrm{Na}}$ presumes that there are three $m$-gates and one $h$-gate that must be open to enable the $\mathrm{Na}^{+}$current to flow via the sodium channel.

This stochastic background of the membrane machinery inspired the development of an alternative class of cardiac cell models in which ion transport is described in the probabilistic terms of Markov processes [4].
Thus, the previous descriptions of the ECG cellular sources can be partitioned into the two separate classes of broadly defined deterministic and stochastic models. To our knowledge, the model of a chaotic BDP in our theory for the first time puts the randomness and determinism in the ECG generation from cellular sources on the common theoretical framework of deterministic chaos. The model not only predicts how the chaotic transient evolves over the time, but also does this with the minimum number of degrees of freedom. Note that $\psi$ and $\kappa$ parameters may be regarded as natural measures of the onset time and amplitude of the mass potential. Therefore, just a single parameter, the $\chi$-factor, defines the shapes of the self-similar element of the ECG. No matter how complex the system of the ion transport, from the viewpoint of the global scale, the chaos factor accumulates dynamic aspects of the microscopic machinery. We qualify this paradigm as the chaotic transformations effect or simply the $\chi$ effect.

The ability of chaotic transformations to accumulate the information from multiple sources into a concise set of parameters may be associated with the presence of the Gaussian distribution in the GMP equation. The normal (Gaussian) distribution is well known as the most important probability distribution in the whole field of probability and statistics. This is because, in accordance with the central limit theorem, any process of random sampling tends to produce a normal distribution of sample values, even if the whole population from which the samples are drawn does not have a normal distribution. A single Gaussian distribution is not suited to account for the temporal changes in the system from which the samples come. In the context of a chaotic BDP, a specific combination of two shifted Gaussian distributions describes how the mean size of the particle population evolves over the time. It is clearly plausible to conclude that standard GMP may be regarded as a time dependent statistical distribution relevant to specific class of chaotic processes.

Our study is in a sharp contrast with the previous approaches to the multi-scale ECG modelling the guiding principles of which presume that extension of the range and number of microscale biophysical and biochemical parameters is a means to reconcile the time evolution of ECG waveform with the underlying cellular machinery. This is a highly under determined task. In reality, the large amount of details and free parameters contained in the model can often obscure rather than illuminate the essentials of the underlying physical processes. The chaotic transformations effect discriminates those aspects of the cellular machinery that are significant on the global scale from those that are not. A crucial deduction from our theory is that mass effect of many micro-scale elements is independent of the physical nature of these elements.

In this context, we may divide GMP parameters into the two main categories: $\kappa$ and $\psi$-physical parameters, $\chi$ - factor - chaos based parameter. $\kappa$ and $\psi$ are physical parameter because they are the measures of physical entities, the voltage and time, respectively. We introduce a special category of a chaos based parameter because the $\psi$-factor 
accumulates both physical measures and statistical variability. As a physical parameter, the $\chi$-factor plays the role of a time constant of the transient potential produced by the deterministic chaos. With regard to statistical issues, the $\chi$-factor accumulates essential aspects of statistical fluctuations at the microscopic scale. To bring the chaos factors into sharper focus, note that transient process in linear dynamic system is defined as the solution of differential or integro-differential equations defined by the physical parameters of the system. By contrast, the $\chi$-factor reflects the chaotic dynamics of the parameters rather that their physical nature. We regard this view as a unique explanation of the fact that self-similar elements identified here in the ECG waveforms replicate the self-similar elements of ERP and eye-blink EMG uncovered in a series of previous studies [11-13].

$\kappa$ and $\psi$ parameters are closely related to conventional ECG measures, the peak amplitudes and latencies. Our theory goes beyond these conventional parameters and suggests a more comprehensive analysis of ECG waveforms using the $\chi$ factor as the measure of the shape for each component identified in the ECG time course. This richer account of the dynamics of the heart electrical activity may provide a basis for a more profound understanding of the ECG, making this already useful measure even more informative in research and clinical settings.

\section{REFERENCES}

[1] R.C.P. Kerckhoffs, S.N. Healy, T.P. Usyk, and A.D. McCulloch, "Computational methods for cardiac electromechanics", Proc. IEEE, vol. 94, pp. 769-783, April 2006.

[2] D. Noble D, "From the Hodgkin-Huxley axon to the virtual heart", J. Physiol., vol. 580, pp. 15-22, April 2007.

[3] R.L. Winslow, S. Cortassa, and J.L. Greenstein. "Using models of the myocyte for functional interpretation of cardiac proteomic data", J. Physiol., vol. 563, pp. 73-81, February 2005.

[4] R.L. Winslow, S. Cortassa, B. O'Rourke, Y.L. Hashambhoy, J.J. Rice, and J.L. Greenstein, "Integrative modeling of the cardiac ventricular myocyte", Wiley Interdisciplinary Reviews: Systems Biology and Medicine, $\mathrm{n} / \mathrm{a}$. doi: 10.1002/wsbm.122, 2010.

[5] D. Noble D, "Modeling the Heart", Physiology, vol. 19, pp. 191197, August 2004

[6] Y. Rudy Y, "Modelling and imaging cardiac repolarization abnormalities", J. Intern. Med., vol. 259, pp. 91-106, January 2006.

[7] J.R. Silva, H. Pan, D. Wu, A. Nekouzadeh, K.F. Decker, J. Cui, N.A. Baker, D. Sept, and Y. Rudy, "A multiscale model linking ion-channel molecular dynamics and electrostatics to the cardiac action potential", Proc. Natl, Acad, Sci. USA, 2009, vol. 106, no. 27 , pp. 11102-6.
[8] K.H.W.J. ten Tusscher, D. Noble, P.J. Noble and A.V. Panfilov, “A model for human ventricular tissue", Am. J. Physiol. Heart Circ. Physiol., vol. 286, pp. H1573-H1589, April 2004.

[9] V. Iyer, R. Mazhari, and R.L.Winslow, "A computational model of the human left-ventricular epicardial myocyte", Biophys. J., vol. 87, pp. 1507-1525, September 2004

[10] S.A. Niederer, M. Fink, D. Noble, and N.P. Smith, "A metaanalysis of cardiac electrophysiology computational models", Exp. Physiol., vol. 94, pp. 486-495, October 2009.

[11] D. Melkonian, E. Gordon, and H. Bahramali, "Single-event-related potential analysis by means of fragmentary decomposition", Biol. Cybern., vol. 85, pp. 219-229, September 2001.

[12] D. Melkonian, T. Blumenthal, and R. Meares, "High resolution fragmentary decomposition - a model based method of nonstationary electrophysiological signal analysis", J. Neurosci. Methods, vol. 131, pp. 149-159, December 2003.

[13] T. Blumenthal, and D. Melkonian, "A model based approach to quantitative analysis of eyeblink EMG responses", $J$ Psychophysiol., vol. 17, pp. 1-11, January 2003.

[14] B. Geselowitz, "On the theory of the electrocardiogram", Proc. IEEE, vol. 77, pp. 857-876, June 1989.

[15] D. Melkonian, and G.K. Kostopoulos, "Stochastic particle formulation of the vesicle hypothesis. Relevance to short-term phenomena", Neuro. Report, vol. 7, pp. 937-942, March 1996.

[16] D.G. Kendall, "On the generalized "birth-and-death" process", Ann. Math. Statist., vol. 19, pp. 1-15, March 1948.

[17] A.T. Bharucha-Reid. Elements of the theory of Markov processes and their applications. NewYork: McGraw Hill, 1960.

[18] A.L. Hodgkin, and A.F. Huxley, "A quantitative description of membrane current and its application to conduction and excitation in nerve", J. Physiol., vol. I I7, pp. 500-544, August 1952.

[19] X. Ning, C. Bian, J. Wang, and Y. Chen, "Research progress in nonlinear analysis of heart electric activities", Chinese Sci. Bull., vol. 51, pp. 385-393, April 2006.

[20] D. Melkonian, E. Gordon, C. Rennie, and H. Bahramali, "Dynamic spectral analysis of event-related potentials", Electroenceph. Clin. Neurophysiol., vol. 108, pp. 251-259, April 1998.

[21] D. Melkonian, "Similar basis function algorithm for numerical estimation of Fourier integrals", Numer. Algor., vol. 54, pp. 73100, May 2010.

[22] R. Bousseljot, D. Kreiseler, and A. Schnabel, "Nutzung der EKGsignaldatenbank CARDIODAT der PTB über das Internet", Biomed. Tech., vol. 40, no. 1, pp. 317, 1995.

[23] D. Kreiseler, and R. Bousseljot, "Automatisierte EKG-Auswertung mit Hilfe der EKG-Signaldatenbank CARDIODAT der PTB", Biomed. Tech., vol. 40, no. 1, pp. 319, 1995.

[24] I. Gussak, P. Bjerregaard, T.M. Egan, and B.R. Chaitman, "ECG phenomenon called the $\mathrm{J}$ wave: history, pathophysiology, and clinical significance", J. Electrocardiol., vol. 28, pp. 49-58, January 1995.

[25] H. Bahramali, D. Melkonian, and O. O'Connell, "Self regulation of the heart: natural frequency and damping of the heart contractions", TOCSJ, vol. 2, pp. 1-10, January 2008.

[26] R. FitzHugh, "A kinetic model of the conductance changes in nerve membrane", J. Cell Comp. Physiol., vol. 66, Suppl 2, pp. 111-117, December 1965 . 